\title{
ERRATUM
}

\section{Endovascular Placement of an Extraluminal Femoropopliteal Bypass Graft in Human Cadavers}

\author{
Manuel Maynar, ${ }^{1}$ Rafael Llorens, ${ }^{1}$ Jesus Uson-Gargallo, ${ }^{2}$ Veronica Crisostomo, ${ }^{2}$ \\ Carmen Lopez-Sanchez, ${ }^{3}$ Virginio Garcia-Martinez, ${ }^{3}$ Zhong Qian, ${ }^{4}$ Jorge Lopera, ${ }^{4}$ \\ Wilfrido R. Castañeda ${ }^{4}$ \\ ${ }^{1}$ Divisions of Vascular Surgery and Endovascular Surgery, Institute of Diagnosis and Minimally Invasive Therapy, Santa Cruz de Tenerife, Spain \\ ${ }^{2}$ Minimally Invasive Surgery Center, Caceres, Spain \\ ${ }^{3}$ Anatomia y Embriología Humana, Facultad de Medicina, Universidad de Extremadura, Apartado 108, 06080 Badajoz, Spain \\ ${ }^{4}$ Division of Vascular and Interventional Radiology, Louisiana State University Health Sciences Center, 1542 Tulane Avenue, New Orleans, LA 70112, USA
}

RE: Cardiovasc Intervent Radiol 28:209-214 (2005). The names of two of the co-authors, Jesus Uson-Gargallo, D.V.M., Ph.D. and
Veronica Crisostomo, D.V.M., Ph.D., were inadvertently omitted from the published version of this article. Their names have been added correctly above. 